\title{
Kernos
}

Revue internationale et pluridisciplinaire de religion grecque antique

$10 \mid 1997$

Varia

\section{L'Hymne homérique à Déméter comme offrande : regard rétrospectif sur quelques catégories de l'anthropologie de la religion grecque}

\section{Claude Calame}

\section{OpenEdition}

\section{Journals}

Édition électronique

URL : http://journals.openedition.org/kernos/652

DOI : $10.4000 /$ kernos. 652

ISSN : 2034-7871

\section{Éditeur}

Centre international d'étude de la religion grecque antique

\section{Édition imprimée}

Date de publication : 1 janvier 1997

Pagination : 111-133

ISSN : 0776-3824

\section{Référence électronique}

Claude Calame, «L'Hymne homérique à Déméter comme offrande : regard rétrospectif sur quelques catégories de l'anthropologie de la religion grecque », Kernos [En ligne], 10 | 1997, mis en ligne le 12 avril 2011, consulté le 19 avril 2019. URL : http://journals.openedition.org/kernos/652 ; DOI : 10.4000/ kernos.652 


\section{L'Hymne bomérique à Déméter comme offrande : regard rétrospectif sur quelques catégories de l'anthropologie de la religion grecque}

Depuis que les relations ritualisées des Grecs avec leurs divinités ont été constituées en « religion », l'étude de cette dernière s'est nourrie à la source de l'anthropologie culturelle et sociale. En effet la comparaison a eu et continue à avoir sur la compréhension des manifestations religieuses dans les communautés civiques hellènes une influence déterminante. L'approche comparative se fonde sur un mouvement sensible dès le début du xvIII ${ }^{\mathrm{e}}$ siècle par exemple chez le Révérend Père Lafitau qui pense trouver dans les coutumes des Indiens d'Amérique du Nord les survivances des premiers stades de développement des cultures de l'Antiquité. Tout en légitimant la comparaison entre premiers Grecs et «sauvages Amériquains », la qualification de «primitif » projette sur un axe temporel, orienté dans le sens de l'épanouissement de la civilisation judéochrétienne, la distance géographique qui sépare les Européens des «autres», barbares vivant dans la lointaine Amérique : l'éloignement dans l'espace (par rapport à l'Europe) vient coïncider avec l'éloignement dans le temps (de l'Occident) $^{1}$. Le relativisme auquel devrait conduire l'approche comparative devient ainsi universalisme!

Mais c'est en particulier dans le contexte de ce qu'on a appelé «l'École de Cambridge » que le comparatisme a pu déployer pour la compréhension des phénomènes relatifs à la religion antique ses effets les plus fertiles. Inspirée notamment par les travaux de J.G. Frazer auquel elle paie une dette explicite de reconnaissance, J.E. Harrison n'hésite pas par exemple à donner une interprétation anthropologique des danses rituelles des Courètes en les assimilant aux rites d'initiation à l'adolescence célébrés par les tribus « primitives » d'Afrique

1 J.-F, LAFITAU, Mœurs des sauvages amériquains comparées aux mours des premiers temps, Paris, 1724, I, p. 3-20, texte mis dans la perspective de l'approche comparative par Ph. BORGEAUD, Le problème du comparatisme en bistoire des religions, in Rev. Europ. Sc. Soc., XXIV. 7 (1986), p. 59-75 (l'ensemble de ce numéro est consacré à la question de la comparaison et du comparatisme); voir également M. DE CERTEAU, Histoire et antbropologie chez Lafitau, in C. BLANKAERT (éd.), Naissance de l'etbnologie? Antbropologie et missions en Amérique, Paris, 1985, p. 62-89. J'aimerais exprimer ma reconnaissance à Pierre Voelke pour son appui dans la mise au point de cette étude. 
noire $^{2}$. Dès lors les historiens de la religion grecque n'ont cessé de trouver une fertile inspiration herméneutique dans le comparatisme.

\section{L'histoire de la religion grecque : un regard rétrospectif}

\subsection{Trois tendances}

Avec tout ce que cet exercice peut comporter de dangers en raison de son caractère en partie arbitraire et de ses résultats nécessairement schématiques, on avancera l'hypothèse que s'esquissent dans la recherche en histoire de la religion grecque de l'après-guerre trois grandes tendances; et l'on cherchera à y associer discrètement en note - exercice encore plus périlleux - quelques titres et, par conséquent, quelques noms. Du côté français d'abord, la méthode structurale telle que l'a défendue et brillamment imposée C. Lévi-Strauss s'est teintée pour la Grèce antique d'une sensibilité à l'aspect social et politique des manifestations religieuses; cette perception était la conséquence de l'intérêt marqué pour une sociologie d'inspiration marxiste. Dans leurs effets simplificateurs, la recherche d'oppositions binaires et l'application de notions telles que le « renversement», «le (radicalement) autre » ou « l'extérieur » ont été tempérées par la conscience des fonctions civiques assumées par des divinités dont champ d'action et modes d'intervention ne se laissent pas réduire à l'unique logique du contraire et du contradictoire $^{3}$. Davantage de fidélité à l'historicisme privilégié du côté italien a entraîné en revanche la recherche germanophone, avec l'impact remarquable qu'elle a eu sur l'autre rive de l'Atlantique, à une attention aux gestes présumés fondateurs de toute religion. Ici, ce sont la connaissance exacte des documents et l'érudition encyclopédique d'un seul savant qui ont corrigé les effets réducteurs d'une théorie historico-génétique inspirée de l'éthologie ${ }^{4}$.

2 Si déjà dans Prolegomena to the Study of Greek Religion, Cambridge, $1908^{2}$, p. XIV, J.E. HARRISON reconnaît sa dette envers les travaux de la religion « primitive » de J. G. FRAZER, c'est essentiellement pour Themis. A Study of the Social Origin of Religion, Cambridge, $1927^{2}$, qu'elle dit trouver son inspiration dans la recherche anthropologique (pour une nouvelle référence à Frazer, voir p. XVII). Davantage encore que dans le Rameau d'Or; J. G. FRAZER a donné toute la mesure de l'éclairage comparatif au bénéfice de grands «mythes » grecs dans l'introduction et dans les appendices de son édition de Apollodorus. The Library, Cambridge Mass.-London, 1921, I, p. IX-XLIII, et II, p. 311-455.

3 On citera pour mémoire les études fondatrices de J.-P. VERNANT, Mythe et sociêté en Grèce ancienne, Paris, 1974, et M. DETIENNE, Les Jardins d'Adonis. La mytbologie des aromates en Grèce, Paris, 1972, avec l'ouvrage commun paru sous le titre Les ruses de l'intelligence. La mètis des Grecs, Paris, 1974, des études qui trouvent souvent leur inspiration dans celles de L. GERNET, réunies sous le titre Antbropologie de la Grèce antique, Paris, 1968, et qui ne sont pas insensibles à celles de J. RUDHARDT, notamment celles réunies dans $D u$ mythe, de la religion grecque et de la comprébension d'autrui (Rev. Europ. Sc. Soc. XIX. 58), Genève, 1981.

4 De W. BURKERT, on lira en particulier Homo Necans. Interpretationen altgriechischer Opferriten und Mytben, Berlin-New York, 1972, avec la thèse reprise dernièrement dans Creation of the Sacred. Tracks of Biology in Ancient Religion, Cambridge Mass.-London, 1996. On n'oubliera pas non 
Sans doute n'est ce pas un hasard si le privilège accordé notamment par les Parisiens à la synchronie a orienté la recherche comparative plutôt du côté des parallèles indo-européens (G. Dumézil), sinon vers ceux offerts par les sociétés tribales contemporaines. En contraste, ce sont les analogies comparatives présentées par les grands textes des cultures mésopotamiennes qui ont séduit les chercheurs en quête de relations de filiation empiriques et diachroniques entre le développement de la religion grecque et les civilisations historiquement susceptibles de l'inspirer. De plus, par sa nature même, l'approche structurale a orienté les premiers davantage vers les textes et donc vers ces récits que l'on appelle depuis la fin du $\mathrm{XVIII}^{\mathrm{e}}$ siècle mythes; indépendamment des fonctions de ces récits, des genres poétiques qui les portent à leur public et des variations que leur fait subir un contexte historique et social en mouvement constant, l'esprit structural a fait qu'on y a recherché, au-delà d'une «mythologie-savoir », soumise aux formes graphiques du changement, une «mythologie-cadre » correspondant à un système de pensée couvrant l'ensemble de la culture grecque ${ }^{5}$. Quant aux seconds, les textes lus dans une perspective davantage historique, sinon génétique, les a rendus davantage sensibles aux pratiques que nous nommons rituels; des pratiques, tels « le sacrifice » ou «l'initiation tribale », que l'interprète estime être actives dans les textes mêmes non seulement parce qu'elles en fourniraient l'occasion, mais surtout parce qu'elles en détermineraient la structure narrative.

Dans une perspective critique ou au contraire plus conciliatrice, des chercheurs hollandais notamment ont su éviter les écueils de ces deux tendances épistémologiques, très marquées, en fournissant des travaux qui ne se plient pas à l'image artificiellement polarisée et forcément simplificatrice présentée ici ${ }^{6}$. De concert avec les travaux d'une tradition anglo-saxonne qui, étrangement, semble avoir mis entre parenthèses pour l'instant toute perspective comparative ${ }^{7}$, leurs

plus, dans une perspective à la fois d'évolution sociale et de comparaison anthropologique, le Paides e Partbenoi, Roma, 1969, d'A. BRELICH et le Saggio sul misticismo greco, Roma, 1965, de D. SABBATAUCCI.

5 Idée développée par M. DETIENNE, La double écriture de la mytbologie entre le Timée et le Critias, in C. CALAME (éd.), Métamorphoses du mythe en Grèce antique, Genève, 1988, p. 17-33 (repris dans L'écriture d'Orphée, Paris, 1989, p. 167-186).

6 Voir la synthèse donnée par J.N. BREMMER dans Greek Religion, Oxford, 1994, et pour la perspective critique en particulier sur la relation entre «mythe » et « rite », H.S. VERSNEL, Inconsistencies in Greek and Roman Religion, 2 vol,, Leiden-New York-Köln, 1990-1993, travaux qui contrastent avec l'effet de doxa parisienne que l'on trouvera dans l'édition française de l'ouvrage, par ailleurs fort bien conçu, de L. BRUIT ZAIDMAN et P. SCHMITT PANTEL, La religion grecque, Paris, 1991. La pudeur calviniste attachée aux liens d'amitié hélvètes me conduit à me limiter à ajouter ici les simples noms de Ph. BORGEAUD et F. GRAF, tout en mentionnant, de manière collective, les travaux conduits dans le cadre de l'institution hôte de ces réflexions, le Centre International d'Étude de la Religion Grecque à Liège.

7 Du côté anglais, on citera volontiers l'ouvrage récent de R. PARKER, Atbenian Religion. A History, Oxford, 1996, et du côté américain, avec une attention portée aux représentations modernes de la religion grecque, les études de A. HENRICHS, parmi lesquelles par exemple "He Has a God in Him." Human and Divine in the Modern Perception of Dionysus, in Th. H. CARPENTER et Ch. A. 
études représentent un troisième mouvement, plus empirique. Dans cette perspective, la religion grecque est désormais devenue un ensemble de pratiques qui, dans le contexte d'une civilisation au profil bien défini, modèlent la vie des Grecs en cité non seulement en réglant les relations des différents représentants de la communauté civique avec leurs héros et leurs dieux, mais qui permettent aussi de développer les aspects les plus symboliques de la culture et de l'idéologie qui en animent l'action sociale.

\subsection{Notions et catégories anthropologiques}

Or l'adoption de la méthode comparative par l'historiographie des manifestations religieuses des Grecs n'a pas uniquement eu un impact décisif pour comprendre les fonctions et les effets de sens de récits ou de pratiques dont le contexte ethnographique fait cruellement défaut. Mais les historiens de la religion hellène n'ont pas hésité à reprendre à l'anthropologie culturelle et sociale la plupart des concepts opératoires qu'elle a créés dans sa visée interprétative, à la fois modélisante et classificatrice ${ }^{8}$. C'est en particulier le cas des notions de "mythe » et de "rite », désormais entrées dans le savoir encyclopédique et partagé de notre propre culture, et maintenant soumises à l'examen critique. Le concept même de «religion » n'échappe d'ailleurs pas à ce mouvement rétrospectif et critique; il serait en effet regrettable qu'il jouisse d'une quelconque immunité 9 ! Au-delà des efforts comparatifs menés dans la distance requise de toute recherche érudite, le recours indispensable à de tels instruments conceptuels n'engage-t-il pas la recherche historique et anthropologique à projeter sur les « religions » antiques nos propres catégories et conceptions ?

Depuis la canonisation du rite et du mythe par leur transformation de catégories analytiques en universaux transcendant les cultures, l'anthropologie culturelle et sociale est devenue plus consciente et plus prudente dans sa relation du soi avec les autres. Fortement dépendante de la tutelle coloniale

FARAONE (éds), Masks of Dionysus, thaca-London, 1993, p. 13-43. Voir aussi les travaux rigoureux de Ch. SOURVINOU-INWOOD, réunis dans "Reading" Greek Culture. Texts and Images, Rituals and Mytbs, Oxford, 1991.

8 À tort ou à raison, C. LÉVI-STRAUSS, Anthropologie structurale, Paris, 1958, p. 386-393, puis D. SPERBER, Le savoir des antbropologues, Paris, 1982, p. 15-48, ont attribué l'un à l'anthropologie, l'autre à l'ethnographie le rôle de la synthèse interprétative; voir à ce sujet les remarques critiques de M.-J. BOREL, Le discours descriptif, le savoir et ses signes, in J.-M. ADAM, M.-J. BOREL, C. CALAME et M. KILANI, Le discours antbropologique. Description, narration, savoir, Lausanne, $1995^{2}$, p. 21-64.

9 La notion de "religion» a fait l'objet, par exemple, des études critiques réunies par J,-B. MARTIN et F. LAPLANTINE (éds), Corps, religion, sociêté, Lyon, 1991; voir également C. BERNAND et S. GRUZINSKI, De l'idolâtrie. Une archéologie des sciences religieuses, Paris, 1988, p. 75-80; T. ASAD, Genealogies of Religion, Baltimore-London, 1993; J. BREMMER, "Religion", "Ritual" and the Opposition "Sacred us. Profane": Notes Towards a Terminological Arcbaeology, in F. GRAF (éd.), Ansichten griechischer Rituale. Festschrift Walter Burkert, Stuttgart-Leipzig, 1997. Sur la genèse des notions de «mythe» et de «rite» je me permets de renvoyer aux nombreuses références que j'ai données dans Mytbe et bistoire dans l'Antiquité grecque. La création symbolique d'une colonie, Lausanne, 1996 , p. 9-46 (voir aussi l'étude citée infra n. 17). 
imposée par l'impérialisme européen sur les sociétés et les cultures différentes, l'enquête ethnographique se soucie désormais d'établir avec les autres une relation dialogique. L'observation distante, prétendue garantie d'objectivité, a fait place à une tentative de constitution intersubjective de la signification ${ }^{10}$. Et quand les informations réunies et négociées dans un dialogue où l'ethnologue accepte de se remettre lui-même en cause sont soumises à la mise en discours qu'implique la forme savante de la monographie, le processus de la «textualisation » est interrogé en termes de traduction transculturelle. En particulier parce que le texte, même élaboré sur une relation d'interlocution, est en définitive destiné à la lecture à distance par un public de formation lettrée et académique, le rapport de l'anthropologue avec une société exotique reste malgré tout marqué par l'asymétrie; une asymétrie constitutive, fondée autant dans la nécessaire différence que dans l'inévitable position de pouvoir de l'Occidental ${ }^{11}$. Cela est d'autant plus vrai de l'anthropologie de la Grèce antique où non seulement les documents à disposition - textes et représentations figurées - sont le produit d'une mise en forme sémiotique et « discursive» préalable, mais où les producteurs de ces manifestations symboliques ne sont évidemment plus en mesure de réagir et de répondre à nos interrogations et à nos sollicitations... À défaut de réels « informateurs », l'historien des religions antiques ne peut plus guère être mis en question que par ses propres collègues !

Or l'immanquable mise en discours qu'implique l'approche anthropologique d'une culture différente et de sa religion - que cette approche soit comparative ou non - est attachée à deux corollaires. D'une part, la transmission d'un savoir passe nécessairement par les procédures du découpage, de l'abstraction et du classement; les contingences de la mise en discours elle-même empêchent la restitution fidèle du vécu dans sa continuité. À cet effet, des concepts opératoires tels le «mythe », le «rite », mais aussi la «magie » le «masque », le «tabou ", le «sacrifice», la « religion» se révèlent aussi précieux qu'ils sont trompeurs. Il s'agit en effet de catégories qui se situent à mi-chemin entre l'empirique et le formel; il s'agit de notions analogues à ces basic-level

10 L'étude que l'on cite en général comme exemple d'anthropologie dialogique et discursive est celle de P. RABINOW, Reflections on Fieldwork in Morocco, Berkeley, 1977 (trad. fr, : Paris, 1988); voir aussi V. Crapanzano, Tubami. Portrait of a Moroccan, Chicago, 1980, et M. TAUSSIG, Sbamanism, Colonialism, and the Wild Man. A Study in Terror and Healing, Chicago-London, 1987. La théorie de l'intersubjectivité dans le dialogisme interprétatif de l'anthropologie a été constituée par F. AFFERGAN, Exotisme et altérité. Essais sur les fondements d'une critique de l'antbropologie, Paris, 1987, p. 225-284.

11 Les processus de textualisation constitutifs de la monographie ont été étudiés en particulier par M. KILANI, Les antbropologues et leur savoir: du terrain au texte, in op. cit. (n. 8), p. 65-100, et L'invention de l'autre. Essais sur le discours antbropologique, Lausanne, 1994, p. 40-62; voir également, du côté américain, C. GEERTZ, Works and Lives. The Antbropologist as Autbor, Stanford, 1988 , p. 1-24 et 129-149. La relation entre dialogisme, asymétric du rapport à l'autre et relativisme culturel a été explorée en particulier par S. BoruTTI, Per un'etica del discorso antropologico, Milano, 1993, p. 95-119; voir maintenant l'essai de synthèse proposé par M. AMORIM, Dialogisme et altérité dans les sciences bumaines, Paris, 1996, p. 21-72. 
categories qui nous permettent non seulement de connaître notre environnement, mais surtout de communiquer à son sujet ${ }^{12}$. Elles jouent un rôle d'autant plus important quand elles s'appliquent à des pratiques et à des résultats de l'activité symbolique qui ne sauraient être séparés des hommes et des communautés qui les ont produits. D'autre part, au caractère forcément flou des catégories semi-empiriques de la pensée anthropologique s'ajoute le fait que toute mise en discours implique une activité du sujet. Quelle qu'en soit la nature psychosociale, ce sujet, compris - comme le suggère É. Benveniste - comme instance de discours, laisse immanquablement dans les textes qu'il a produits les traces de son activité d'énonciation. La distanciation de qui parle ou écrit à l'égard de son discours est aussi illusoire que l'objectivité de l'observateur vis-àvis du produit de son observation ${ }^{13}$.

Cela signifie que l'historien de la religion grecque qui trouve son inspiration interprétative dans la lecture comparative des travaux des anthropologues doit opérer un retour critique aussi bien sur les notions classificatoires floues qui en articulent l'organisation sémantique, que sur les effets énonciatifs de leur mise en forme discursive. Cela signifie surtout que, confronté dans sa recherche essentiellement à des textes et à des images, ce même historiographe doit se montrer réceptif autant aux catégories indigènes qui les traversent qu'aux traces laissées par leur mise en discours. Cette double recommandation semble s'imposer avec d'autant plus de force en histoire des religions de l'Antiquité que les plus riches parmi les documents à disposition se présentent sous la forme de poèmes. Il s'agit donc des manifestations d'un processus de production symbolique complexe; leur forme poétique indique par elle-même qu'elles ont une visée pragmatique essentielle. Dans une performance qui correspond en général à un acte de culte, ces textes poétiques donnent autant d'indications sur l'histoire des dieux et des héros auxquels il s'adressent que sur les pratiques dont ils dépendent. C'est ce que l'on aimerait montrer dans une étude (partielle) portant sur la partie finale de l'Hymne homérique à Déméter.

12 Définies dans une perspective cognitiviste par G. LAKOFF, Cognitive Semantics, in U. ECO, M. SANTAMBROGIO et P. VIOLI (éds), Meaning and Mental Representations, Bloomington-Indianapolis, 1988, p. 119-154 (voir aussi Women, Fire, and Dangerous Things. What Categories Reveal About the Mind, Chicago, 1987, p. 269-303), les catégories du niveau de base aident par exemple à saisir la nature de ces classifications pratiques et semi-empiriques que sont les taxinomies de genres poétiques : $c f . \mathrm{J}$. MOLINO, Les genres littéraires, in Poétique, 93 (1993), p. 3-28.

13 Voir É. Benveniste, Problèmes de linguistique générale, Paris, 1966, p. 258-266, avec les prolongements proposés et les références données quant au repérage des traces de l'énonciation portées par tout discours dans C. CALAME, Le récit en Grèce ancienne. Énonciations et représentations de poètes, Paris, 1986, p. 11-29, et Pour une sémiotique de l'énonciation : discours et sujet, in M. COSTANTINI et I. DARRAUlT-HARRIS (éds), Sémiotique, pbénoménologie, discours; du corps présent au sujet énonçant, Paris, 1996, p. 137-154. En ce qui concerne les procédures de l'énonciation dans le discours anthropologique, on renverra aux différentes contributions publiées dans ADAM, BOREL, CALAME et KILANI, op. cit. (n. 8). 


\section{L'Hymne à Déméter entre «mythe » et « rite »}

Dans une lecture de la fin de l'Hymne à Déméter sensible aux indices de la mise en discours du poème, c'est sur un usage critique des catégories du «mythe » et du « rite » que l'on aimerait centrer ce bref exercice d'anthropologie de la religion antique. On doit à $\mathrm{J}$. Harrison une première tentative réflexive à ce propos, par la substitution aux désignations traditionnelles de termes repris au grec : legomenon et drômenon. Mais dans la mesure où il ne s'agit que d'une substitution et où ces termes sont effectivement employés par les Grecs dans l'Antiquité tardive uniquement (pour nous, Pausanias) pour être restreints au domaine des cultes à mystères, les notions introduites par $\mathrm{J}$. Harrison ne font que reproduire les catégories entrées dans le savoir partagé des anthropologues. Le legomenon serait ce qui est dit sur l'agi, drômenon: le mythe en tant que discours narratif et traditionnel serait le commentaire d'un rite compris comme action collective et réglée; d'un côté la parole, de l'autre l'acte, la première venant a posteriori justifier le second ${ }^{14}$.

Les contours flous des catégories à peine évoquées ont eu pour conséquence de multiplier les tentatives de définition, destinées à rester insatisfaisantes. On renoncera à les passer en revue. Il suffira donc pour l'instant de noter que les manifestations qu'il est coutume d'appréhender sous les notions de « mythe » et de « rite » correspondent régulièrement en Grèce à des pratiques de culte et qu'elles dépendent dans leur ensemble de ce que l'on peut grossièrement dénommer le «processus symbolique ». À l'aide de moyens sémiotiques différents, les «rites » se révèlent être aussi spéculatifs que les « mythes » et les seconds ont une dimension performative qui les rapprochent des premiers. Les uns comme les autres sont le résultat, pour parler en terme de poétique grecque, d'une activité de «mimésis » qui laisse une large marge à la création fictionnelle, sans perdre pour autant de son « efficacité ${ }^{15}$.

14 J. HARRISON, Themis. A Study of the Social Origin of Religion, Cambridge, $1927^{2}$, p. $29,42-45$ et 328-331, avec la mise au point historique que j'ai proposée à ce propos dans Thésée et l'imaginaire athénien. Lègende et culte en Grèce antique, Lausanne, $1996^{2}$, p. 20-27; voir également W.G. DOTY, Mythography. The Study of Myths and Rituals, Tuscaloosa-London, 1986, p. 41-78, et VERSNEL, op. cit. (n. 6), II, p. 15-88.

15 Encore dernièrement, par une nouvelle enquête sur cet objet anthropologique privilégié qu'est devenu le naven, « rite» accompli de manière multiforme sur les bords du Sépik de Papouasie-Nouvelle Guinée, M. HOUSEMAN et C. SEVERI, Naven ou le donner à voir, Essai d'intepprétation de l'action rituelle, Paris, 1994, p. 183-202, ont montré que, actes symboliques, les « rituels» permettaient en particulier de s'interroger sur les relations sociales tissées dans le contexte des systèmes rivaux du kinship et du parentbood; ils engendrent dans cette mesure une "représentation collective des enjeux de l'identité ». Cela implique que, contrairement à ce qu'a voulu affirmer D. SPERBER, Le symbolisme en général, Paris, 1974, p. 141-157, le « rite » (en tant que «croyance ») est producteur de significations, mais par des moyens sémiotiques en général motivés, bien différents de ceux de la langue! Sur le « rituel " comme représentation, voir aussi M. BLOCH, Prey into Hunter. The Politics of Religious Experience, Cambridge, 1992, p. 1-7, à propos des pratiques collectives marquant les différentes étapes de la vie de l'individu. 


\subsection{Les fonctions énonciatives du macarismos}

Comme c'est le cas d'autres Hymnes bomériques, l'Hymne à Déméter trouve sa conclusion dans la formule du "macarismos", familière de cette poésie d'action qu'est la poésie mélique. Plus exactement, redoublé, ce macarismos, assure la transition de l'epica laus aux preces dans un hymne articulé, comme pratiquement toutes les compositions du corpus des Hymnes bomériques, en trois parties : évocation de la divinité, éloge descriptif et narratif plus ou moins développé de ses qualités, adresse directe sous forme de prière ${ }^{16}$. Le récit de l'enlèvement de Perséphone par Hadès s'achève avec l'intervention de Déméter. D'une part, la déesse rétablit sur terre la fécondité des labours. D'autre part, elle fait aux souverains d'Éleusis la démonstration de l'accomplissement de pratiques rituelles si respectables qu'on ne doit ni les transgresser, ni s'en informer de manière indue, ni les divulguer; des pratiques et peut-être des objets ( $\delta \rho \eta \sigma \mu o \sigma u ́ v \eta$ i $\epsilon \rho \hat{\nu} \nu$ et ő $\rho \gamma\llcorner\alpha$ ) marqués par la crainte religieuse et le silence ( $v$, 470-479) que l'on a naturellement et un peu rapidement identifiés avec ceux qui régnaient sur les mystères d'Éleusis de l'époque classique ${ }^{17}$.

Le macarismos faisant suite à cette phase de sanction du récit, qui débute lui-même au v. 2, est donc double. Le premier vante le bonheur (ő $\lambda$ เos) de qui a vu ces pratiques sacrées ( $l \epsilon \rho a ́$ ) susceptibles de conférer à son bénéficiaire une part ( $\mu$ oîpa, aîa) nouvelle même lorsqu'il aura disparu dans les brumes souterraines ( $v, 480-482$ ); le second exalte la félicité (ő $\lambda$ เos) du mortel qui, aimé des deux déesses, voit s'installer à son foyer Ploutos, l'incarnation de la prospérité

16 Le caractère spécifique de la structure tripartite des Hymnes bomériques a été bien redéfini par J. M. BREMER, Greek Hymns, in H.S. VERSNEL (éd.), Faith, Hope, and Worship. Aspects of Religious Mentality in the Ancient World, Leiden, 1981, p. 193-215, avec les précisions que j'ai cru devoir apporter dans Variations énonciatives, relations avec les dieux et fonctions poétiques dans les Hymnes homériques, in $M H, 52$ (1995), p. 2-19. Dans son remarquable commentaire de l'Hymne, N. J. RICHARDSON, The Homeric Hymn to Demeter; Oxford, 1974, p. 313-314, donne un série de parallèles à l'emploi de la forme du macarismos dans la poésie archaíque; on y ajoutera ALCMAN, fr. 1, 37-38 et 15 Davies, avec les références complémentaires que j'ai données dans Alcman. Introduction, texte critique, témoignages et commentaire, Roma, 1983, p. 323 et 355-356.

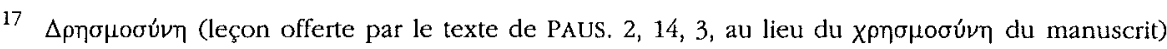
et ő $\rho \gamma$ la sont des termes qui, désignant en général l'action de type rituel, ressortiront après l'époque classique spécifiquement au domaine des cultes à mystères : $c f$. RICHARDSON, op. cit. (n. 16), p. 251 et 303-304, ainsi que C. CALAME, "Mythe " et "rite» en Grèce: des catégories indigènes ?, in Kernos, 4 (1991), p. 179-204, et A. MOTTE et V. PIRENNE-DELFORGE, Le mot et les rites. Apercu des significa-

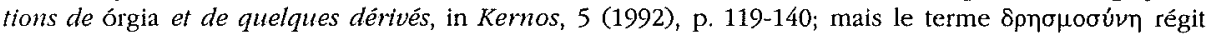
ici $\iota_{\text {có }}$ qui, dans la poésie homérique, peut avoir le sens très général de "rites sacrés » ou d'« objets sacrés ": voir J. CASABONA, Rechercbes sur le vocabulaire du sacrifice en grec des

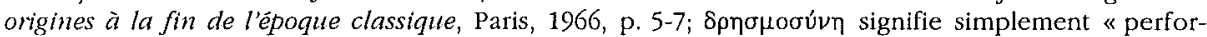

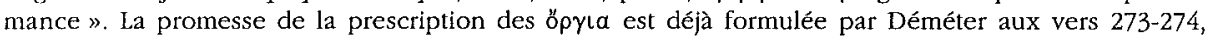
avec un probable jeu étymologisant sur ce terme qui est rapproché du verbe $\epsilon^{\prime} \rho \delta \omega$, « faire »: cf. J. STRAUSS ClAY, The Politics of Olympus. Form and Meaning in the Major Homeric Hymns, Princeton, 1989 , p. 241-243. Le silence et le secret qui entouraient les pratiques d'Éleusis sont pour nous attestées pour la première fois par la profanation dont on a accusé Alcibiade en 415: cf. ANDOC., Myst., 29-33; voir aussi, quelques années plus tard, SOPH., OC 1050-1053; autres références, plus tardives, chez RICHARDSON, op. cit. (n. 16), p. 308-310. 
agricole (v. 487-489) ${ }^{18}$. La promesse pour qui jouit de la protection des deux déesses en raison de sa participation aux « rites » est donc double, portant sur la vie de mortel et sur la vie future; une promesse que l'emploi de ö $\lambda \beta$ ios au lieu de $\mu a ́ k a \rho$ réfère à un bonheur de mortel et non pas à un processus d'immortalisation. Les deux macarismoi sont séparés l'un de l'autre par la fin de la phase de sanction du long récit qui couvre l'ensemble de l'Hymne: Déméter et Coré se rendent sur l'Olympe où elles habitent désormais, vénérées et respectées, aux côtés de Zeus. Il s'agit bien de la contre-partie divine des effets positifs pour les hommes de l'heureuse conclusion du rapt de Perséphone. Il ne reste dès lors plus au locuteur - aède ou rhapsode - qu'à interpeller en termes cultuels les deux déesses pour leur demander à son tour, en échange de son chant et avec la promesse d'un nouveau chant, les ressources de la vie (ßíotos, v. 490-495) : do ut $\operatorname{des}^{19}$.

D'un point de vue comparatif, on remarquera à ce propos qu'aussi bien dans l'Hymne bomérique 25 destiné à Apollon et aux Muses que dans le prologue de la Théogonie qui, adressé aux Muses, présente la même structure qu'un Hymne bomérique, la transition entre louange descriptivo-narrative de la divinité et prière est également assurée par un macarismos ${ }^{20}$. Dans ces deux cas la formule généralisante, comme dans le second macarismos de l'Hymne à Déméter (v. 486-487), vante le bonheur d'être aimé par la divinité précisément

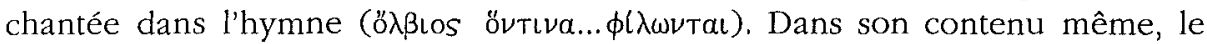
macarismos ne fait donc qu'annoncer en termes généraux l'échange proposé par le locuteur / narrateur dans la prière finale. Le contrat de réciprocité proposé aux dieux est d'autant plus marqué dans l'Hymne 25 qu'Apollon et les Muses, loués en tant que protecteurs des aèdes, sont les divinités mêmes susceptibles d'inspirer le chant qui leur est offert en retour!

18 Comme parallèles à ce double macarismos, on mentionne habituellement les deux formules semblables trouvées chez PIND., fr. 137 Maehler, et SOPH., fr. 837 Radt, et situées par chacun des auteurs qui citent ces vers (Clément d'Alexandrie et Plutarque) dans le contexte des mystères d'Éleusis : cf. M. CANNATÀ FERA, Pindarus. Threnorum Fragmenta, Roma, 1990, p. 204-209; on remarquera néanmoins que ces deux macarismoi poétiques se réfèrent uniquement au bonheur connu après la mort par les initiés. Ici, l'insistance sur l'intervention de Ploutos (le fils de Déméter et de Iasion chez HÉs., Théog., 969-974) qui confère aux mortels une prospérité essentiellement agricole montre que ce bonheur touche les protégés de Déméter et de Perséphone déjà dans cette vie même : chez HÉs., Trav., 170-173, l'ő $\lambda \beta$ os dont jouissent les héros vivant dans les Îles des Bienheureux est aussi attaché à des récoltes abondantes puisque trisannuelles : voir M.L. WEST, Hesiod. Works and Days, Oxford, 1978, p. 193-194, et RICHARDSON, op. cit. (n. 16), p. 313-314 et 316-321.

19 Le terme $\beta$ lotos, comme $\beta$ los ou le personnage de Ploutos, renvoie à une abondance et une prospérité surtout agricoles; voir par exemple HÉs., Trav., 31-32, 42, 232, etc., avec les références données infra n. 32. La structure d'échange marquant la prière qui achève la plupart des Hymnes bomériques a été analysée par BREMER, art. cit. (n. 16), p. 196-197, et par CAlAME, art. cit. (n. 16), p. 8-12.

20 Hhom., 25, 4-5 (voir aussi 30, 7-8, avec un macarismos bien développé) et HÉs., Théog., 96-97; sur la structure et la fonction du proème assumées par le prélude de la Théogonie, voir l'op. cit. (n. 13), p. 43-47 et 60-62, avec les références données dans l'art. cit. (n. 16), p. 19 n. 40. 
L'analyse linguistique et énonciative concourt à confirmer fortement le rôle de transition du macarismos. En effet si le dernier acte de Déméter vis-à-vis des souverains d'Éleusis auxquels elle révèle les rites marqués du secret est formulé à l'aoriste ( $\delta \epsilon \hat{\imath} \xi \epsilon$, v. 474), la formulation du premier macarismos est dominée par le

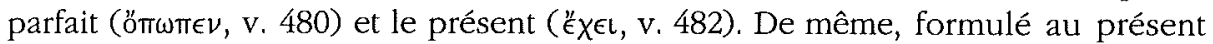

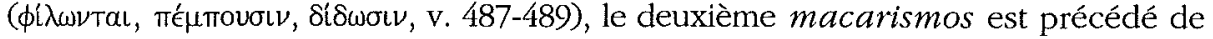
la mention de l'ultime action de la déesse qui s'en est allée rejoindre sur

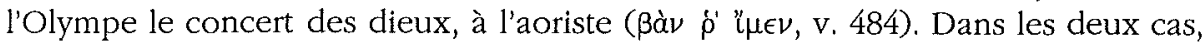
la transition entre les temps du récit et la validité atemporelle du macarismos est marquée par un énoncé gnomique au présent; ces énoncés rappellent, respectivement, l'effet pour les mortels de la piété inspirée par les deux divinités (elles retiennent la voix des hommes: loxável, v. 479), et leur séjour auprès de Zeus, vénérées de tous (vaıєtáovoı, v. 485). Aux deux formulations généralisantes du macarismos et de l'assertion qui les introduit succèdent les formes impératives

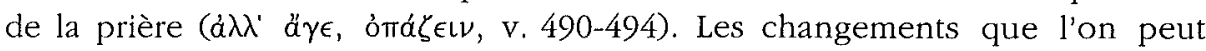
constater dans les protagonistes des actions verbales signalées suivent ce même mouvement: noms propres des divinités comme objets de l'action narrative, pronoms d'une troisième personne généralisante pour les bénéficiaires des macarismoi et finalement, au vocatif, les noms des divinités apostrophées par le locuteur implicitement présent dans les formes de l'impératif.

Il faut donc attendre le terme extrême de la composition hymnique pour voir apparaître le locuteur / narrateur : il se manifestera en tant qu'é $\gamma u ́$ dans l'ultime vers du poème. Formulaire, cette promesse d'évoquer ( $\mu \nu$ rooual, v. 495) dans un autre chant encore le destinataire de l'hymne engage la fonction pragmatique, sinon performative de l'ensemble de la composition. On y reviendra en conclusion. Pour l'instant, à partir de cette ouverture conclusive du présent sur un futur intentionnel, l'articulation entre passé narratif et présent gnomique, souvent interprétée en terme de «mythe » et de « rite », nous convie à revenir au récit, jusqu'ici à peine évoqué. S'agit-il d'un récit étiologique et, en prenant appui sur cette qualité, est-il légitime de voir dans sa logique des analogies avec le déroulement des pratiques cultuelles qu'il est censé fonder?

\subsection{Un « mythe » d'initiation?}

Les efforts n'ont donc pas manqué pour détecter dans les différents épisodes du long récit qui constitue l'essentiel de l'Hymne à Déméter les reflets des pratiques qu'il devrait légitimer. Successivement, la recherche par Déméter de sa fille à la lumière des torches (v. 47-48) a été mise en relation avec le "drame mystique », également éclairé par des torches, qui est censé constituer le centre des mystères d'Éleusis; le jeûne de neuf jours marquant pour Déméter ce moment de deuil $(v$. 49-50) a permis de reconstruire l'imposition aux initiés d'Éleusis d'une pratique analogue; la double épiphanie de Déméter quand elle franchit le seuil du palais de Célée puis quand elle révèle son identité à Métanéira (v, 188-190 et 275-280) pourrait correspondre à l'expérience vécue par 
les initiés eux-mêmes durant les mystères; et la tentative d'immortalisation de Démophoon a été comprise comme le mythe de fondation de l'initiation mystique $^{21}$. L'addition de ces éléments d'étiologie pourrait donc donner à penser que l'Hymne à Déméter n'est que le legomenon du drômenon par excellence que constitue le déroulement rituel des mystères d'Éleusis.

En raison même du point de vue adopté dans les travaux antérieurs de l'auteur de la présente étude, on aimerait aborder la question de la projection des catégories du «mythe » et du « rite » sur la relation étiologique du texte avec des pratiques de culte en se concentrant sur les interprétations initiatiques qu'on a pu proposer de l'Hymne à Déméter. La mise en discours de la présente étude entend donc s'insérer dans une perspective dialogique et réflexive.

À la suite des propositions formulées naguère par $\mathrm{H}$. Jeanmaire, les historiens de la religion grecque tentés par l'anthropologie n'ont pas tardé à trouver dans les nombreux récits qui, en Grèce ancienne, mettent en scène jeunes filles et jeunes gens le fameux schéma ternaire qui a permis à A. van Gennep, à partir de nombreuses manifestations rituelles, de définir une nouvelle catégorie anthropologique : le «rite de passage », fondé sur la séquence «rites de séparation - rites de marge - rites d'agrégation » et spécifié en « rite d'initia tion tribale » quand la transition concerne des adolescents passant à l'âge adulte $^{22}$. C'est ainsi que, plus récemment, on a pu affirmer à propos du récit de l'enlèvement de Coré : «while I have certain reservations as to the applicability of the van Gennep model to rites of female initiation, (...) it does seem to fit the

21 Voir successivement et dans l'ordre CLÉM. ALEX., protr. 2, 12, 2 (cf. RICHARDSON, op. cit. [n. 16], p. 162 et 165-167); M. P. NILSSON, Griechiscbe Feste von religiöser Bedeutung, Leipzig, 1906, p. 321; RICHARDSON, op. cit. (n. 16), p. 208-209 et 256; D. SABBATUCCI, Saggio sul misticismo greco, Roma, 1965 , p. 163-165, ainsi que P. SCARPI, Letture sulla religione classica. L'inno omerico a Demeter, Firenze, 1976, p. 199-218. D'autres exemples sont mentionnés par RICHARDSON, op. cit. (n. 16), p. 24-30, et par STRAuss CLAY, op. cit. (n. 17), p. 202-205. À toutes ces tentatives étiologiques, il faut naturellement ajouter la purification, l'abstinence, l'aischrologie et la consommation du $\kappa u k \in \omega \nu$ qui marquent le deuil de la déesse (v. 192-211) : cf. RICHARDSON, op. cit. (n. 16), p. 211-218. Sur l'HDém. en général, voir F. WEHRLI, Die Mysterien von Eleusis, in ARW, 31 (1934), p. 77-104, qui y voit des relations étiologiques fortes entre «mythe » et « rite », G. SFAMENI GASPARro, Miti e culti mistici di Demetra, Roma, 1986, p. 29-45, et K. CLINTON, Myth and Cult. The Iconography of the Eleusinian Mysteries, Stockholm, 1992, p. 28-37 et 96-99, qui entend désormais mettre l'Hymne en relation avec le festival des Thesmophories d'Athènes : cf. infra n. 50.

22 H. JEANMAIRE, Couroi et Courètes. Essais sur l'éducation spartiate et sur les rites d'adolescence dans l'antiquité bellénique, Lille, 1939, p. 296-307, part d'une interprétation de la tentative d'immortalisation de Démophoon comme « rite de passage par le feu» et comme épreuve initiatique pour mettre brièvement en relation le rapt de Perséphone avec une "période d'initiation » et finalement voir dans les mystères, "forme éleusinienne des Thesmophories», un complexe combinant des « rites agraires » et des « rites de clôture» du passage entre enfance et adolescence; quant à l'« anodos » de Coré, il serait célébré à l'occasion des Thesmophories (p. 268-282; cf. infra $\mathrm{n}$. 50). Plus prudemment A. VAN GENNEP, Les rites de passage, Paris, 1909, p. 125-131, porte son attention sur les mystères d'Éleusis eux-mêmes dans lesquels il voit des "rites d'initiation » faisant "passer le néophyte du monde profane au monde sacré » (pour la définition du schéma tripartite du rite de passage, voir p. 19-33). Pour des lectures en termes initiatiques de différentes manifestations narratives et cultuelles, voir par exemple les contributions publiées par A. MOREAU (éd.), L'initiation, 2 vol., Montpellier, 1992. 
Persephone myth extremely well ${ }^{23}$. Ce sont autant les termes de model et de fit qui orientent cette assertion que ceux de rite et de $m y t h$ : il s'agit non seulement de soumettre la logique du récit de la descente aux Enfers de Perséphone à un modèle, mais aussi de plaquer sur cette manifestation narrative un schéma élaboré à partir de pratiques à caractère rituel. Dans cette perspective, l'enlèvement de la jeune fille par Hadès, le viol supposé par le maître des Enfers et finalement son retour auprès de sa mère et des dieux de l'Olympe sont interprétés comme les trois phases d'un rite d'initiation : enlèvement / ségrégation, perte de la virginité / «betwixt and between », accession à l'Olympe / agrégation.

\subsection{Temps et espace narratifs}

Une lecture attentive à la logique du récit et aux valeurs qu'il met en jeu révèle qu'il est porté par un langage narratif assez différent de celui, rituel, de l'initiation tribale. Du point de vue des marqueurs temporels et de la logique qui en découle, le récit de l'Hymne bomérique à Déméter se signale par une contradiction patente : en situant l'enlèvement de Coré sur une prairie fleurie, le début du récit correspond avec le moment du printemps que son terme, et donc sa sanction, prévoit au contraire pour le retour de la jeune fille sur la terre. Cette étrange coïncidence temporelle entre la saison de la disparition de Perséphone sous terre et celle de son retour parmi les mortels et les dieux a donné passablement de fil à retordre à ses interprètes. En se fondant sur l'exégèse indigène qui a rapidement assimilé le séjour infernal de quatre mois imposé chaque année à Perséphone, avec son retour printanier, au cycle saisonnier dessiné par la germination puis la croissance du blé, on a lu dans le récit de l'enlèvement de la jeune fille une allégorie du retour du printemps; cette saison s'incarnerait dans une «grain maiden » qu'un jeu de mot sur la dénomination Koúp a bien vite hellénisée ${ }^{24}$. Et pour résoudre l'immanquable contradiction entre un printemps qui est marqué dans le récit par la disparition de Coré sous terre et l'association à la même saison du retour et de la renaissance de la jeune fille (v. 401), on a imaginé de faire coïncider le séjour narratif de Coré auprès d'Hadès non plus

23 B. LINCOLN, The Rape of Persephone: A Greek Scenario of Women's Initiation, in HTbR, 72 (1979), p. 223-235, avec la critique de l'application au récit (en particulier à celui du rapt de Coré) du schéma de l'initiation formulée par R. L. FOWLER, Greek Magic, Greek Religion, in ICS 20 (1995), p. 122; voir aussi W. BURKERT, op. cit. (n. 4), p. 289-292. Présentée au X Congrès de la FIEC à Québec (août 1994), l'étude de B. ZWEIG, The Rape of Persepbone and Ancient Rituals of Female Initiation, va dans le même sens.

24 "Since the Mother was the grain mother, the Daughter must have been the grain maiden »: C. KERÉNYI, Eleusis. Arcbetypal Image of Motber and Daughter, Princeton, 1967, p. 130-133; voir déjà HARRISON, $o p$. cit. (n. 2), p. 271-276: Déméter et Coré en tant que «Corn-Mother » et "CornMaiden », ainsi que JEANMAIRE, op. cit. (n. 22), p. 270-272. De telles assimilations sont proposées dès l'Antiquité par les poètes comiques EUBOULOS, fr. 75, 10 et ANTIPHANÈs, fr. 55, 8-9 Kassel-Austin : voir également PORPH., fr. 358F 1-17 Smith cité par EUSĖBE, Prép. Év., 3, 11, 9; autres références chez

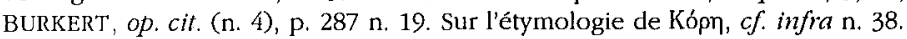


avec les mois d'hiver, mais avec l'été, saison où, en Méditerranée, le grain moissonné est censé être gardé dans des contenants souterrains ${ }^{25}$. Quel que soit le réalisme de cette hypothèse, elle explique d'autant moins la coïncidence établie par le texte entre la saison du rapt de Perséphone et celle fixée pour son retour annuel qu'en particulier dans les pays méditerranéens, le blé germe déjà en automne.

Force est dès lors de dissocier la logique du récit de celle du rythme des saisons; force est de reconnaître que le second substitue une temporalité cyclique à la temporalité linéaire du premier : dans le récit, Perséphone disparaît dans les Enfers une année entière ( $c f$. v. 305 !) pour les quitter au printemps; dans le cycle annuel, elle ne séjourne que quatre mois dans la demeure d'Hadès. C'est ici le lieu de rappeler, à propos du cycle des saisons, qu'à l'époque archaïque on distinguait trois périodes : un bref printemps, un long été que l'on disait parfois se poursuivre dans la période de maturation de fruits de l'ótrópa, et un hiver d'environ quatre mois. Le tiers du temps imposé à Perséphone pour son séjour annuel et cyclique dans le sein de la terre auprès d'Hadès (v. 398-400) correspond donc bien à un hiver qui se distingue nettement des deux ou trois autres périodes consacrées à l'activité agricole ${ }^{26}$. À cette identification qui, proposée dès l'Antiquité, situe l'entrée annuelle de Perséphone dans l'Hadès à la fin de l'automne, il convient d'ajouter que, de toute façon, le récit ne saurait représenter le «mythe » de fondation de l'institution des saisons, préexistantes comme l'indique d'emblée l'évocation de Déméter ápnфópos (v. 54, 192 et 492;

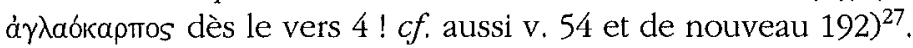

Face à cette absence d'homologie entre la logique temporelle, supposée initiatique, du récit et la temporalité « rituelle » que semble sinon instituer, en tout cas confirmer sa sanction, on peut tenter de passer de sa dimension temporelle à l'espace qu'il dessine. Le lieu du rapt de Perséphone correspond à l'une de ces grasses prairies émaillées de fleurs si souvent offertes au regard dans la légende grecque; tendre gazon et fleurs printanières évoquent le désir amoureux suscité par la beauté des jeunes filles qui les animent de leurs danses et de leurs

25 Il s'agit de l'hypothèse formulée par M. P. NILSSON, Die Eleusiniscben Gottbeiten, in ARW, 32 (1935), p. 79-141 (republié dans Opuscula selecta II, Lund, 1952, p. 542-623, en particulier p. 577-581) et en partie reprise par BURKERT, op.cit.(n. 4), p. 287-288.

26 Pour la répartition de l'année agricole en trois ou quatre saisons de longueur inégale, voir ALCMAN, fr. 20 Davies, avec le commentaire donné dans l'op. cit. (n. 16), p. 374-376, ainsi que les références citées par RICHARDSON, op. cit. (n. 16), p. 284-285, notamment sur les auteurs qui, dès l'Antiquité, ont identifié le séjour de Coré dans les Enfers avec l'hiver (voir aussi supra n. 24).

27 On verra à ce sujet les remarques prudentes de STRAUSS CLAY, op. cit. (n. 17), p. 254-256, et celles, décisives, formulées par J. RudHardT, A propos de lHymne homérique à Déméter, in $M H, 35$ (1978), p. 1-17 (repris dans l'op. cit. [n. 3l, p. 227-244), sans oublier le commentaire de H.P. FOLEY, The Homeric Hymn to Demeter. Translation, Commentary, and Interpretive Essays, Princeton, 1993, p. 5859. 
jeux $^{28}$. Sans l'aide du moindre geste de culture, Gaia y fait croître le narcisse à l'odeur envoûtante qui va séduire la jeune fille de Déméter, dans un acte qui interpelle mortels et immortels et qui sollicite dans un mouvement cosmologique ciel, terre ( $\gamma \alpha \hat{i} \alpha)$ et mer (v. 8-14; voir v. 33-35 et 38).

Le contraste est dès lors entier avec la terre ( $\chi \theta \dot{\omega} \nu, v .16)$ qui ouvre sa béance sous les pieds de la jeune fille dès qu'elle tend ses mains pour cueillir le narcisse odorant. Enlevée par Hadès, le frère de Zeus et de Déméter elle-même, Coré est désormais plongée dans les ténèbres brumeuses des entrailles de la terre (v. 7780). Tout au long du séjour de sa fille sous terre, Déméter quant à elle jouit sur terre de l'hospitalité des mortels, dans la demeure du souverain d'Éleusis, Céleus, et de son épouse Métanéira. Après la mise en échec, par l'ignorance des mortels (v. 256-257), de la tentative d'immortalisation du petit Démophoon, le fils des souverains d'Éleusis, et après l'installation de la déesse dans le temple que ceuxci édifièrent en son honneur, la stérilité qui frappe le sol de la terre (v. 305-309) marque le début d'une nouvelle étape spatiale. Pour empêcher l'extinction de la race des mortels par la famine (v. 310-312), Zeus décide de libérer Perséphone de son séjour infernal. Le retour de la jeune femme se déroule en deux moments : retrouvailles avec Déméter dans le temple terrestre d'Éleusis (v. 384-385), puis accession de la mère et probablement de la fille à l'Olympe où est institué pour Perséphone le partage annuel de son séjour entre les brumes obscures de la demeure d'Hadès et la compagnie lumineuse des Immortels (v. 445-447; cf. v. 398-400 et v. 463-465) 29

$\mathrm{Si}$, du point de vue spatial, la narration se limite à mettre en scène pour Déméter un séjour prolongé dans la demeure d'hommes mortels avant de réintégrer la déesse au lieu qu'elle a quitté, le parcours suivi par Perséphone peut apparemment se prêter à une interprétation en termes de passage : surface terrestre - domaine infernal - Olympe. Néanmoins, dans la mesure où elle n'est pas définitive, la transition n'a rien d'initiatique. Ce n'est que pour les deux tiers du cycle annuel que la jeune Perséphone est réintégrée au domaine des dieux olympiens; pour le tiers restant, elle reste attachée à l'espace qui pourrait correspondre à celui, marginal, de la mort initiatique. Cette ambivalence spatiale

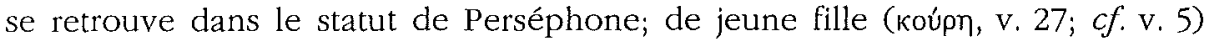
éveillant le désir d'un homme adulte quand elle danse avec ses compagnes sur un pré fleuri, Coré devient l'épouse (ăkoıtıs, v. 79 et 343; cf. v. 363) d'Hadès, mais

28 À ce propos voir en dernier lieu les distinctions que j'ai tenté d'introduire dans L'éros dans la Grèce antique, Paris, 1996 , p. 183-186, mais aussi, indépendamment des considérations historicistes, toutes les références données par 1 . CHIRASSI, Elementi di culture precereali nei miti e riti greci, Roma, 1968, p. 91-124.

29 Même si le texte des v. 441-443 n'est pas explicite, RICHARDSON, op. cit (n. 16), p. 295-296, admet qu'à l'issue du récit et Déméter et Perséphone rejoignent le ciel (cf. v. 336-338). Sur la répétition des vers (en partie lacunaires) indiquant l'attribution à Perséphone d'un double séjour, cf. 1 bid., p. 300-301. Le rôle étiologique de l'épisode de Démophoon est expliqué en particulier par R. PARKER, The Hymn to Demeter and the Homeric Hymns, in $G \& R, 38$ (1991), p. 1-17. 
tout en restant la fille de Déméter (v. 363, 387-388, 406, 445, etc.). Consacré par le cycle qui marque le double séjour de la jeune femme, ce partage entre le statut de femme adulte vivant avec son époux aux Enfers et celui de la jeune fille attachée à sa mère en sa demeure olympienne est d'autant plus frappant que les «noces » de Perséphone sont célébrées durant la période qui devrait correspondre à la phase de marge de tout rite d'initiation destiné aux adolescents. Si le déroulement de la cérémonie du mariage telle qu'elle est conçue dans l'Athènes classique semble suivre, du point de la jeune fille, la logique attribuée au rite d'initiation tribale, Perséphone ne connaît ni le détachement définitif de la demeure de son père et de son statut de vierge, ni l'agrégation entière à la maison et à l'autorité du mari à qui elle doit donner une descendance ${ }^{30}$.

Dans les transformations de valeurs qu'elle organise du point de vue temporel, spatial et social, la logique du récit du rapt de Perséphone tel qu'il est raconté dans l'Hymne bomérique à Déméter ne correspond donc pas à la séquence qui articule les unes avec les autres les différentes phases du rite de passage, et plus précisément du rite d'initiation tribale. Ni pour la fille, ni pour la mère, il n'y a de véritable passage; il n'y a ni pour l'une, ni pour l'autre d'accession au statut entièrement nouveau et définitif que présuppose le rite d'initiation.

\subsection{Entre agriculture et mortalité}

Pour en revenir aux valeurs attachées aux espaces mis en scène par l'intrigue du récit de l'Hymne à Déméter, la narration les organise effectivement en un avant et un après. Au début du récit, la prairie couverte de fleurs printanières évoquant le pouvoir d'éros pour offrir aux jeunes filles l'aire de danse propre à leurs jeux adolescents (v. 5-14); en son terme, les fruits produits par une terre dûment labourée, mais aussi les feuilles et les fleurs qui la couvrent (v. 471 473); et dans la période intermédiaire, le grain caché sous terre (à l'instar de Perséphone !) qui menace par la famine de détruire la race des hommes (v. 305311, 332 et 351-354). Du point de vue des figures assumées par la temporalité du récit, Coré disparaît au moment de la floraison printanière pour réapparâtre sur terre non pas après quatre mois, mais au terme de l'année de stérilité et de famine inspirée par la colère de Déméter (v. 305-306). Le retour de Coré et la fin de la colère de sa mère sont marqués non seulement par la réapparition des tendres épis dans les gras sillons de la plaine de Raros, mais aussi par la renaissance de la végétation correspondant avec le développement du prin-

30 Le déroulement de la cérémonie du mariage classique peut être lu en termes initiatiques : voir les références données dans l'op. cit. (n. 28), p. 126-130. Pour une interprétation du récit du rapt de Coré comme institution du mariage dans sa composante patriarcale, voir SCARPI, op. cit. (n. 21), p. 109-137, avec la critique de cette hypothèse formulée par LINCOLN, art. cit. (n. 23), p. 227-228, qui préfère voir dans la perte de la virginité de Perséphone aux Enfers un trait caractéristique de la période de séclusion d'un processus initiatique; sur la relation du récit de Perséphone avec le mariage, voir aussi FOLEY, op. cit. (n. 27), p. 79-83 et 104-112. 


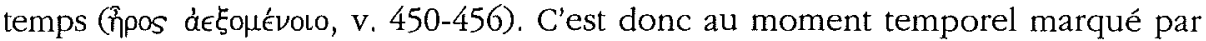

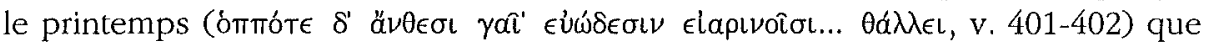
coïncident la logique linéaire du récit du rapt de Perséphone et la logique cyclique de la répartition de l'année entre un séjour hivernal dans le monde des morts et un séjour olympien, correspondant à la longue saison de croissance, de maturation et de récolte des fruits de la terre.

Du point de vue narratif, l'attachement du retour de Perséphone à la floraison printanière reçoit une confirmation dans la reprise par la jeune fille ellemême du récit de son propre enlèvement, au moment de ses retrouvailles avec Déméter (v. 406-433). L'évocation de la prairie inspirant le désir et des fleurs printanières qui accueillent les danses des Océanides suscitant elles-mêmes le désir amoureux y tient une place de choix, en écho à la croissance au printemps des fleurs odorantes qui marquera le retour cyclique de la jeune fille (v. 417-428 en écho aux vers 401-402). Tout se passe en somme comme si, narrativement, la structure annulaire que dessine le récit de Perséphone avec le début de la narration de l'Hymne assurait le passage de la logique linéaire du «mythe » à la temporalité cyclique du « rite».

De plus, dans sa colère, Déméter ne se limite pas à cacher sous terre le grain source de vie et de prospérité pour les hommes mortels; mais, par ce geste, elle prive également les dieux olympiens de l'hommage rayonnant des offrandes et

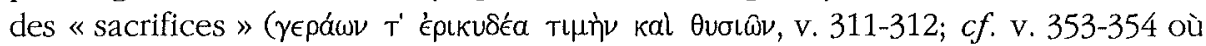
Déméter est dite anéantir les honneurs dus aux immortels, ainsi que v. 331-332 où la déesse prive simplement l'Olympe de sa présence) ${ }^{31}$. L'analogie est frappante avec le début du récit de Pandôra tel qu'il est narré dans Les Travaux et les Jours d'Hésiode. Cette version de la fabrication et du don aux hommes de la jeune fille attachée aux fatigues et aux maladies qui sont le lot des mortels se déroule - rappelons-le brièvement - selon la séquence suivante: les dieux cachent aux hommes le $\beta$ los, les privant ainsi des ressources de la vie que les vers précédents identifient avec les fruits de Déméter et dont les mortels, dans l'état antérieur, disposaient sans travailler (v. 42-46; cf. v. 31-32); en raison de la ruse de Prométhée, que le récit parallèle de la Théogonie (v. 535-560) permet d'identifier avec le premier sacrifice sanglant, Zeus dans sa colère cache un élément qui va se révéler être le feu (v. 47-50); Prométhée dérobe le feu au bénéfice des hommes (v. 50-52); par l'intermédiaire d'Épiméthée, Zeus impose alors aux mortels qui se nourrissent de pain le mal que représente Pandôra, sous les apparences d'un don (v. 53-89). Le résultat, en structure annulaire, reprend, en la modifiant, la situation de départ : alors que les hommes vivaient auparavant sur la terre sans

31 Les gúotal, que l'on traduit communément par «sacrifices», correspondent à des offrandes, notamment végétales, dont on manie ou consomme la partie restante: voir J. RUDHARDT, Notions fondamentales de la pensée religieuse et actes constitutifs du culte dans la Grèce classique, Genève, 1958, p. 249-253, et CASABONA, op. cit. (n. 17), p. 125-131. Pour le sens de tupry en tant qu'honneur rendu à une divinité, $c f$. RICHARDSON, op. cit. (n. 16), p. 260-261. 
les peines et les maladies qui condamnent au trépas, ils sont désormais en proie aux maux jaillis de la jarre ouverte par Pandôra (v. 90-105) ${ }^{32}$.

Tout en tentant d'éviter l'abus méthodologique qui consisterait à inscrire la logique du récit de l'Hymne à Déméter dans celle du récit hésiodique, on ne peut s'empêcher de voir dans le premier l'exposition du développement qui, préalable, est présupposé par le second. Sans doute dans la narration des Travaux les difficultés posées par le passage de l'état général, où les Immortels cachent le ßíos aux hommes, à l'état singulier où Zeus leur cache le feu peuventelles s'expliquer par la nécessité qu'impose la structure annulaire : indiquer d'emblée, au début ( $v .42-46$ ), ce qui sera la situation d'aboutissement du récit (v. 90-92 et 100-104) ${ }^{33}$. Il n'en reste pas moins que, dans la représentation grecque de la civilisation, l'institution de la culture céréalière semble précéder celle du sacrifice animal. Quoi qu'il en soit, du point de vue du régime alimentaire garantissant le développement civilisé de la condition humaine, la prospérité attachée à la production des fruits de Déméter est complémentaire du partage des viandes animales réglé par la pratique du sacrifice sanglant ${ }^{34}$.

Mais, comme on l'a relevé, ce que l'Hymne à Déméter raconte, dans la perspective étiologique qui traverse tout Hymne bomérique, ce n'est pas l'institution de la culture céréalière selon le rythme des saisons. En effet, dans le renouvellement de son dépit et de sa colère, après l'échec de sa tentative d'immortaliser Démophoon, Déméter, en cachant le grain, annule l'effet fertilisant des labours, préexistants; elle bloque la production des fruits de la terre, privant du même coup les dieux des tıนal offertes par les hommes (v. 305-312 et 351 353). Or ce sont précisément de nouvelles rıนal que Zeus offre à Déméter pour tenter de l'associer à nouveau au monde des dieux qu'elle vient de quitter : « les privilèges qu'elle voudrait choisir au sein des dieux immortels » (v. 328). Mais ces Tı́ai s'avèrent en définitive concerner davantage la fille que la mère qui, dans sa seconde épiphanie, se présente en tant que turáoxos (v. 268). Dans la bouche

32 A l'analyse structurale du «mythe prométhéen " tentée par VERNANT, op. cit. (n. 3), p. 177194, qui présente le désavantage de superposer les deux versions hésiodiques du récit en réduisant des divergences précisément significatives, on préférera celle offerte par le même auteur dans M. DETIENNE et J.-P. VERNANT, La cuisine du sacrifice en pays grec, Paris, 1979, p. 37-132.

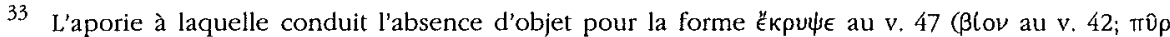
au v. 50) est exposée par WEST, op. cit. (n. 18), p. 155-156; voir l'analyse narrative du récit de Pandôra que j'ai proposée dans Le proème des Travaux d'Hésiode. Prélude à une poésie d'action, in F. BLAISE, P. JUDET DE LA COMBE, Ph. ROUSSEAU (éds), Les métiers du mythe: Lectures d'Hésiode, Lille, 1996, p. $169-189$.

34 «La culture céréalière est donc la contrepartie du rite sacrificiel, son envers » et, plus précisément, "se nourrir de plantes domestiques cultivées, d'animaux domestiques sacrifiés, tels sont donc les deux aspects solidaires d'un régime d'alimentation qui établit (l'homme) dans le statut intermédiaire qui définit ses conditions propres d'existence », peut conclure VERNANT, op. cit. (n. 32), p. 60 et 63, sur la base d'une étude qu'il limite aux récits hésiodiques. On verra aussi les réflexions assez spéculatives sur le régime alimentaire décrit dans le récit de l'HDém. chez SCARPI, op. cit. (n. 21), p. 73-108. Sur agriculture et représentation grecque de la civilisation, voir F. HARTOG, Mémoire d'Ulysse. Récits sur la frontière en Grèce ancienne, Paris, 1996, p. 107-109 et 144-147. 
d'Hadès, les fonctions privilégiées accordées par Zeus correspondent en effet au pouvoir que le souverain des Enfers assure à Perséphone sur tous les êtres vivants, à l'occasion de son séjour dans le monde d'en bas; elles correspondent aussi aux dons et pratiques sacrificielles que lui devront les mortels (v. 364-369). Dans la bouche de Déméter, l'honneur accordé à sa fille consiste dans le partage annuel de son séjour entre Enfers et Olympe (v. 397-400). L'attribution de cet honneur est naturellement du ressort de Zeus qui le transmet à la mère pour le destiner à sa fille (v, 441-447; message répété par Rhéia aux v, 460-465) ${ }^{35}$. Dans les différentes versions de la légende, la distribution et l'attribution aux dieux de leurs domaines et fonctions propres sont en général le corollaire de l'établissement du pouvoir du père des dieux ${ }^{36}$.

Tout le long récit de l'Hymne bomérique à Déméter contribue donc à définir et établir le statut et la fonction de Perséphone auprès de sa mère Déméter. L'allure même prise par la formule initiale d'evocatio non seulement associe d'emblée la fille à la mère, mais, par le relatif hymnique engageant la laus epica, elle focalise toute la narration sur Perséphone ${ }^{37}$. Quant au statut que définit pour elle le récit, Coré, en correspondance avec la résidence partagée qui lui est assignée, se trouve être en alternance une femme adulte (en tant qu'épouse d'Hadès) et une jeune fille (en tant que fille de sa mère) : à la fin du

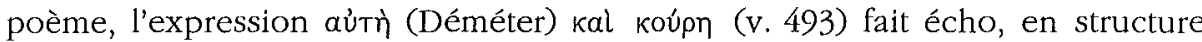

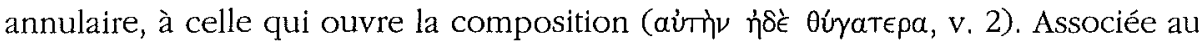
printemps, Perséphone assume auprès de Déméter l'agricultrice la fonction complémentaire de susciter dans les entrailles de la terre la germination, la croissance et la floraison à laquelle l'attache son statut de jeune fille, inscrit dans sa dénomination en tant que Koúpn, Coré ${ }^{38}$. Mais, par l'alternance qui règle son séjour, la vierge-épouse permet aussi la communication et des dieux et des mortels avec le domaine d'en bas, sur lequel règne Hadès ${ }^{39}$.

35 Sur les lacunes que présente le texte des v. 397-400 et sur la répétition du message de Zeus aux v. 460-465, voir le commentaire de RICHARDSON, op. cit. (n. 16), p. 283 et 300-301. L'institution préexistante du rythme des saisons et de l'agriculture est soulignée par les interprètes cités supra n. 27.

36 Voir en particulier HÉs., Théog., 71-74, et les différents textes mentionnés par RUDHARDT, art. cit. (n. 27), p. $2-7$.

37 J'ai repris la question de la structure et du rôle de la partie introductive des Hymnes bomériques dans l'art. cit. (n. 16), p. 6-8, en donnant de nombreuses références bibliographiques à ce propos.

38 Rappelons qu'étymologiquement kóp fait référence à la croissance et que, par métaphore, kópos désigne le rejeton d'une plante : cf. P. CHANTRAINE, Dictionnaire étymologique de la langue grecque. Histoire des mots, Paris, 1968, p. 567-568. Pour la dénominatin de Coré, voir RICHARDSON, op. cit. (n. 16), p. 140 et 170.

39 Comme cela a été fort bien montré par RUDHARDT, art. cit. (n. 27), p. 8-12, qui attribue abusivement à Déméter « la croissance de la végétation» (p. 9), et par STRAuSS CLAY, op. cit. (n. 17), p. 211-213 et 255-257, dont on s'étonne néanmoins qu'elle puisse affimer « the threefold division of the year corresponds to the tripartite division of the cosmos " : conformément à la bipartition du 
Pour formuler en des termes encore plus généraux les fonctions conjuguées de Déméter et de Coré dans leur domaine d'action commun, on constatera que le récit, en se focalisant sur la relation entre la mère et la fille auxquelles cèdent successivement les représentants du pouvoir «patriarcal » que sont Hadès et Zeus, place tout le processus civilisateur de l'agriculture sous la protection des femmes alors que le sacrifice sanglant est essentiellement affaire d'hommes. À cet égard, l'intervention finale et inhabituelle de Rhéia comme messagère de Zeus auprès de Déméter est particulièrement significative. En transmettant à sa fille l'offre faite par le souverain de l'Olympe à Perséphone (v. 441-447 et 459466), elle place la déesse de l'agriculture dans le même statut de filiation vis-à-vis

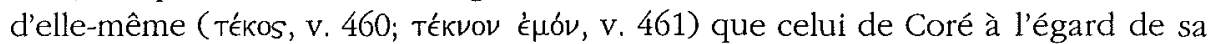
mère Déméter ${ }^{40}$. Les affinités que, dans la représentation grecque de la civilisation humaine, le genre (entendu comme gender) féminin entretient avec la culture agricole et l'alimentation végétarienne se marquent dans l'extraordinaire déploiement des relations métaphoriques régulièrement établies entre céréaliculture, mariage, reproduction et courotrophie. Dans l'Hymne lui-même, l'épisode de Démophoon en porte témoignage.

Mais, dans ce monde sans animaux, le travail protégé par Déméter consacre aussi le caractère mortel de la condition humaine. Si l'Hymne à Déméter a bien une fonction étiologique, c'est moins par une relation de correspondance terme à terme entre «mythe » et « rite » que dans l'institution des grandes représentations constitutives, par le processus symbolique, d'une idéologie.

\section{Au-delà de l'aition, l'acte de culte}

Dans l'Hymne à Déméter, c'est bien de mortalité dont il s'agit principalement. Entre le monde d'en bas contrôlé par Hadès, désormais accompagné de Perséphone, et l'Olympe dominé par Zeus, auprès duquel siège à nouveau sa sœur Déméter, il y a la surface terrestre, cultivée par les hommes mortels pour assurer leur subsistance au cours de leur vie limitée. C'est précisément auprès de ces hommes, dans son temple (v. 384-386) puis dans la plaine de Raros (v. 450), que Déméter retrouve sa fille libérée des Enfers, avant de se rendre avec elle sur l'Olympe ( $v, 483-486)$ : le temps de faire à nouveau croître le grain dans la plaine éleusinienne dûment labourée et surtout de donner aux maîtres mortels de l'endroit ses instructions (v. 470-479). En effet, aux tı $\mu$ al imparties par Zeus correspondent les tua dues par les hommes; et à la promesse faite par Hadès à

séjour de Perséphone, l'Hymne distingue simplement le domaine où dieux et mortels communiquent régulièrement (ciel, terre, mer) du monde d'en bas.

40 Le caractère central de la relation mère-fille dans le récit de l'Hymne a été souligné par FOLEY, op. cit. (n. 27), p. 80 et 118-137, qui sous-estime cependant le rôle joué par Rhéia (p. 131-132). Pour une analyse en termes de gender voir N. CHODOROW, Family, Structure and Feminine Personality, in M. ROSALDO et L. LAMPHERE (éds), Women, Culture and Society, Stanford, 1974, p. 4366, étude reprise dans FOLEY, op. cit. (n. 27), p. 243-265. 
Perséphone de «sacrifices » et de cadeaux constamment offerts par les hommes (v. 365-369) correspond, au terme de la narration, l'institution par Déméter des actes de culte sacrés en l'honneur des deux déesses (v. 475-479).

Dès lors s'éclaire la fonction qu'assume sur le plan sémantique le double macarismos assurant la transition entre ce terme de l'epica laus narrative et les preces qui concluent l'hymne. Cette fonction est en elle-même double : d'une part, le premier macarismos établit pour l'ensemble du genre humain la possibilité, par la vision des actes sacrés révélés par Déméter, d'une extension de

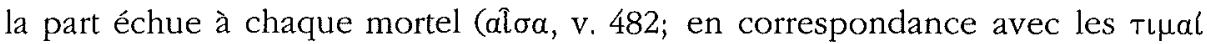

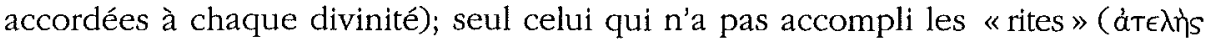
i $€ \omega \hat{\nu}, v .481$ ) sera privé d'un destin s'étendant dans le royaume brumeux que désormais, aux côtés d'Hadès, domine aussi Perséphone ${ }^{41}$. D'autre part, comme on l'a dit, la seconde formule assure à chaque mortel qui le veut bien la prospérité et la richesse agricoles rétablies par Déméter et désormais dispensées par les deux déesses, réunies sur l'Olympe (v. 483-489).

Du point de vue syntaxique et énonciatif, le double macarismos transpose dans l'énoncé même du poème l'indéniable fonction étiologique de la narration. Par la forme générale de "on-vérité » assumée par ces deux séries de vers, c'est la voix de l'aède qui prend en charge les effets de l'institution par Déméter de sa propre célébration éleusinienne, avec les valeurs que le récit a construites. Le locuteur se fait le porte-parole des conséquences pratiques, pour l'ensemble des mortels habitant sur terre ("̋s... '̇ de l'accomplissement des actes de cultes prévus par les dieux ${ }^{42}$. Par cet intermédiaire énonciatif, le poème devient lui-même acte de culte.

Cette fonction cultuelle de l'Hymne à Déméter et de la narration qui en constitue l'essentiel est pleinement confirmée par l'invocation directe et la prière qui concluent le poème. Ce que le locuteur demande aux deux déesses de

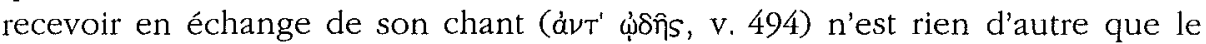
ßlotos qu'elles accordent à ceux qui ont accompli les « rites»; cette correspondance est soulignée en particulier par la reprise d'un terme formé sur prophron-aux v. 487 et 494 . L'exécution même du poème représente donc l'un

41 STRAuSs ClAY, op, cit. (n. 17), p. 261-265, insiste sur le fait que ce premier macarismos établit une nouvelle relation entre vie et mort; voir aussi la bonne étude de L.L. ALDERINK, Mytbical and Cosmological Structure in the Homeric Hymn to Demeter, in Numen, 19 (1982), p. 1-16. Pour nous, ce n'est à vrai dire qu'à partir de l'époque classique que les termes composés sur té̉os peuvent désigner plus spécifiquement les rites d'initiation et s'appliquer en particulier aux mystères d'Éleusis: voir les références données par RICHARDSON, op. cit. (n. 16), p. 314. Sur le sens général de Tà Lepá comme "objets sacrés " ou "rites sacrés», cf. supra n. 17. La reprise dans le premier

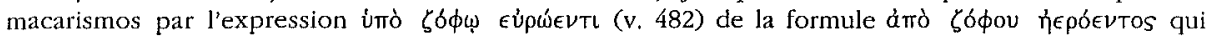
caractérise le domaine d'Hadès (v. 80,337, 402 et 406) est un indice de sa valeur étiologique.

42 Du début à la fin, l'Hymne à Déméter est traversé par l'opposition et la complémentarité entre mortels et immortels. La tentative (étiologique) de fusion des deux sphères culmine au centre de la narration, avec la préparation du кuкєúv et la tentative d'immortalisation de Démophoon (v. 210-254) : voir à ce propos STRAUSS CLAY, op. cit. (n. 17), p. 235-240. 
de ces actes propres à susciter la prospérité. Ainsi, en tant que proème, l'Hymne bomérique adressé à Déméter et à Perséphone ne se limite pas à introduire un

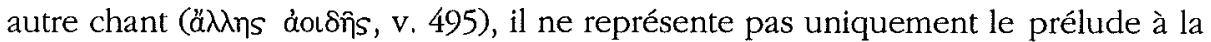
récitation d'une autre composition épique ${ }^{43}$; mais, en tant qu'acte de culte, il insère cette réalisation poétique dans la séquence des lepá et des ő $\gamma\llcorner\alpha$ dont il fait lui-même partie. La demande d'intervention directe adressée aux déesses dans sa conclusion évoque la double épiphanie de Déméter racontée dans la partie narrative. L'hymne comme chant de culte propose aux dieux l'échange de bienfaits institué en réciprocité dans la révélation des gestes accomplis à Éleusis.

Le récit en partie étiologique que déroule l'hymne chanté n'est donc pas le legomenon du drômenon qui serait fondé dans et par la narration ${ }^{44}$; il l'est d'autant moins que, quelle que soit la relation de l'Hymne à Déméter avec les mystères d'Éleusis, le drômenon est aussi un deiknumenon. Mais la narration est elle-même un drômenon, sinon un deiknumenon. L'application des catégories du «mythe » et du « rite» qu'on attache à ces dénominations d'inspiration hellène ne font que brouiller la fonction et la logique du récit et du chant qui le porte : dans sa fonction rituelle, la narration du rapt de Perséphone échappe à la notion $\mathrm{du}$ «mythe » entendu par exemple comme «traditional tale with secondary, partial reference to something of collective importance ${ }^{45}$; de plus, dans sa logique narrative, son intrigue ne répond pas au schéma tripartite du rituel de l'initiation tribale.

Faire du récit du rapt de Perséphone tel qu'il est réalisé dans l'Hymne bomérique à Déméter un "mythe », c'est effacer sa dimension non seulement pragmatique, mais aussi «performative ${ }^{46}$ : en exécutant ce poème et en nar rant

43 La fonction des Hymnes bomériques en tant que proèmes visant à insérer les récitations de poèmes homériques dans une célébration cultuelle particulière est décrite en particulier par F. CÁsSOLA, Inni omerici, Milano, 1975, p. XII-XXI, ainsi que par M. COSTANTINI et J. LALLOT, Le mpooípiov est-il un proème?, in Études de littérature ancienne III. Le texte et ses représentations, Paris, 1987, p. 13-27; autres références chez CALAME, art. cit. (n. 16), p. 2-4 et 17-19.

44 On sait que l'étтotrela formait le centre rituel de l'initiation éleusinienne : références chez RICHARDSON, op. cit. (n. 16), p. 26-30 et 310-314; on en a souvent retrouvé un écho textuel dans la

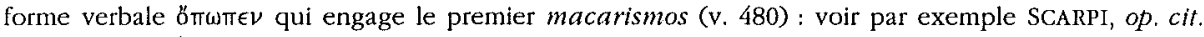
(n. 21), p. 42-46, et PARKER art. cit. (n. 29), p. 12-13.

45 Selon la définition proposée par W. BURKERT, Structure and History in Greek Mytbology and Ritual, Berkeley-Los Angeles-London, 1979, p. 23, et adaptée en particulier par J. BREMMER, What is a Greek Myth?, in J. BREMMER (éd.), Interpretations of Greek Mytbology, London-Sydney, 1987, p. 1-9, et par F. GRAF, Greek Mytbology. An Introduction, Baltimore-London, 1993, p. 1-8. Voir aussi les précisions apportées par W. BURKERT lui-même dans Mytbos. Begriff, Struktur, Funktionen, in F. GRAF (éd.), Mythen in mythenloser Gesellschaft. Das Paradeigma Roms, Stuttgart-Leipzig, 1993, p. 9-24, et par R. BuXTON, Imaginary Greece. The Contexts of Mytbology, Cambridge, 1994, p. 9-17.

46 Par référence aux réflexions de BENVENISTE, op. cit. (n. 13), p. 267-276, je désigne par performatifs les énoncés qui sont par eux-mêmes des actes (de parole); dans le cas de la poésie grecque cette notion implique une part de «mimésis» dans la performance au sens large où l'entend par exemple G. NAGY, Poetry as Performance. Homer and Beyond, Cambridge, 1996, p. 5386. Sur les aspects performatifs de la narration des «mythes", on verra les études réunies par P.-Y. 
cette histoire, l'aède accomplit un acte de culte qui, du point de vue spatial, tend à faire coïncider la résidence olympienne des deux déesses, la demeure infernale de Perséphone et le lieu d'intervention terrestre de Déméter avec le lieu où il chante lui-même - Éleusis (v. 490). Mais faire de ce même récit un « rite d'initiation », c'est oblitérer le dynamisme de sa logique : du point de vue temporel, la récitation du poème fait déboucher le déroulement linéaire de la narration non seulement sur le cycle annuel dont Perséphone est désormais la maîtresse, mais aussi sur la réitération cultuelle dont le caractère formulaire du texte - dans sa structure et dans son langage - est lui-même l'indice ${ }^{47}$. La «ritualisation » du récit que nous appréhendons comme «mythe » va même si loin que, dans la dimension spatiale comme dans la dimension temporelle, le bic et nunc de la narration s'ouvre sur des lieux voisins et sur un futur prochain : à côté d'Éleusis, Paros dans les Cyclades et Antrôn en Thessalie (v. 490-491), deux lieux de culte consacrés à Déméter à l'époque homérique ${ }^{48}$; et au terme du poème présent, la promesse d'un autre chant ( $\mu \nu$ ńooual, v. 495) dédié à la divinité. Le double macarismos, dans sa formulation généralisante, ne fait qu'accentuer ces virtualités de réitération (rituelle !) pour appeler la présence de la divinité.

L'énonciation de l'Hymne bomérique à Déméter fonctionne donc comme acte de culte; mais de quel culte? Cette ultime question découle de l'autre versant d'une conception où le récit, tout en assumant une fonction étiologique, ne saurait représenter le simple legomenon du drômenon. Si la référence constante du poème au culte rendu à Déméter par les Éleusiniens est indéniable, les indices textuels de datation (toujours relative en tradition orale) se combinent avec l'absence de toute mention d'Athènes pour conduire l'interprète à une période où Éleusis n'était pas encore intégrée au territoire de l'Attique ${ }^{49}$. Et tout porte à croire qu'en cette probable fin du $\mathrm{VII}^{\mathrm{e}}$ siècle, non seulement les mystères d'Éleusis n'étaient pas encore intégrés au calendrier officiel de la cité d'Athènes, mais que le culte éleusinien rendu à Déméter et à Coré, s'il était peut-être déjà constitué en "rite d'initiation » comme pourrait l'indiquer l'emploi de áte $\lambda$ ńs (v. 481), partageait des traits nombreux avec le festival panhellénique des

JACOPIN (éd.), La parole mytbique en Amérique du Sud (Bull. Soc. Suisse Americ. 57/58), Genève, $1993 / 1994$.

47 Ch. SEGAL, Orality, Repetition and Formulaic Artistry in the Homeric "Hymn to Demeter", in C. BRIllante, M. CANTIlena, C. O. PAVESE (éds), I poemi epici rapsodici non omerici e la tradizione orale, Padova, 1981, p. 107-160, fournit une analyse fouillée des effets de sens du style formulaire de l'Hymme; voir en particulier les p. 151-157 sur le rôle joué dans le poème par le parfum des fleurs et la grenade.

48 Pour le culte rendu à Déméter à Paros et à Antrôn, voir les indications données par RICHARDSON, op. cit. (n. 16), p. 321-322.

49 La question controversée de la datation relative de l'Hymne à Déméter en rapport avec l'institution athénienne des mystères d'Éleusis est reprise par RICHARDSON, op. cit. (n. 16), p. 5-12, et par CÁsSOLA, op. cit. (n. 43), p. 31-33; pour des arguments d'ordre linguistique, voir RICHARDSON, op. cit. (n. 16), p. 30-65 et 331-338, ainsi que R. JANKO, Homer, Hesiod and the Hymns. Diacbronic Development in Epic Diction, Cambridge, 1982, p. 181-183. 
Thesmophories; ceci dans la célébration des limites de la mortalité des hommes en relation avec leur mode d'alimentation et avec les statuts assignés aux femmes ${ }^{50}$. Tenter de le montrer devra faire l'objet d'une autre étude.

Pour l'instant, on se contentera de la conclusion soufflée, à distance et dans une approche qui s'est voulue énonciative et dialogique, par les notions indigènes en confrontation avec les nôtres : en tant que poésie chantée, considérée dans le processus de sa mise en discours, le «mythe » en Grèce archaïque et classique est « rite».

Claude Calame

Université de Lausanne

Section des Sciences de l'Antiquité

BFSH 2

CH - 1015 LAUSANNE

50 Tout récemment, K. CLINTON, The Autbor of the Homeric Hymn to Demeter, in OAth, 16 (1986), p. 43-49, et op. cit. (n. 21), p. 28-37 et 96-99, a émis l'hypothèse que les aitia de 'Hymne à Déméter se référaient non pas aux mystères d'Éleusis, mais aux Thesmophories, notamment dans leur version athénienne; l'hypothèse avait déjà été avancée par JEANMAIRE, op. cit. (n. 22), p. 268-282. Mais la transmission des ŏ $\rho$ la aux souverains d'Éleusis et la destination des macarismoi au genre humain, femmes et hommes, rendent néanmoins difficile une référence étiologique à un festival réservé, à Athènes, aux seules femmes! La reprise du même argument dans The Sanctuary of Demeter and Kore at Eleusis, in N. MARINATOS et R. HÄGG (éds), Greek Sanctuaries. New Approacbes, London-New York, 1993, p. 110-124, ne m'a pas convaincu; voir aussi à ce sujet PARKER, art. cit. (n. 29), p. 6 avec n. 22, ainsi que FOLEY, op. cit. (n. 27), p. 169-178. 